\title{
Homoclinic Points in Symplectic and Volume-Preserving Diffeomorphisms
}

\author{
Zhihong Xia ${ }^{1}$ \\ Department of Mathematics, Northwestern University, Evanston, Illinois 60208, USA
}

Received: 6 March 1995/Accepted: 28 July 1995

\begin{abstract}
Let $M^{n}$ be a compact $n$-dimensional manifold and $\omega$ be a symplectic or volume form on $M^{n}$. Let $\phi$ be a $C^{1}$ diffeomorphism on $M^{n}$ that preserves $\omega$ and let $p$ be a hyperbolic periodic point of $\phi$. We show that generically $p$ has a homoclinic point, and moreover, the homoclinic points of $p$ is dense on both stable manifold and unstable manifold of $p$. Takens [11] obtained the same result for $n=2$.
\end{abstract}

\section{Introduction and Statement of Main Results}

Let $M^{n}$ be an $n$-dimensional compact manifold with a symplectic or volume structure. Recall that a volume structure is a non-degenerate differential $n$-form $\omega$ on $M^{n}$ and a symplectic structure is a closed differential two-form $\omega$ such that $\omega \wedge \cdots \wedge \omega(n / 2$ times $)$ is a non-degenerate volume form. A symplectic manifold is always even dimensional. We consider diffeomorphisms of $M^{n}$ that preserve the differential form $\omega$. A diffeomorphism that preserves symplectic (resp. volume) form $\omega$ is called a symplectic (resp. volume-preserving) diffeomorphism. Symplectic diffeomorphism arises naturally as a time-one map and the Poincare map of Hamiltonian systems.

Let $\operatorname{Diff}_{\omega}^{r}\left(M^{n}\right)$ denote the set of $C^{r}$ diffeomorphisms that preserve $\omega$, with uniform $C^{r}$ topology. Let $\phi \in \operatorname{Diff}_{\omega}^{r}\left(M^{n}\right)$, then $\phi^{*}(\omega)=\omega$. Let $p$ be a point in $M^{n}, p$ is said to be a periodic point of $\phi$ with period $k$ if $\phi^{k}(p)=p$. Periodic points with period one are called fixed points. A periodic point $p$ with period $k$ is said to be hyperbolic if $\left.d\left(\phi^{k}\right)\right|_{T_{p}\left(M^{n}\right)}: T_{p}\left(M^{n}\right) \rightarrow T_{p}\left(M^{n}\right)$ has no eigenvalue on the unit circle. For any hyperbolic periodic point $p$, there exist a stable manifold, denoted by $W_{\phi}^{s}(p)$ and an unstable manifold, denoted by $W_{\phi}^{u}(p)$. A homoclinic point of $p$ with respect to $\phi$ is an intersection of $W_{\phi}^{u}(p)$ and $W_{\phi}^{s}(p)$, which differs from $p$, i.e., $q$ is a homoclinic point of $p$ if $q \in W_{\phi}^{s}(p) \cap W_{\phi}^{s}(p) \backslash\{p\} . q$ is a transversal homoclinic point of $p$ with respect to $\phi$ if $T_{q}(M)=T_{q}\left(W_{\phi}^{s}(p)\right) \oplus T_{q}\left(W_{\phi}^{u}(p)\right)$.

We state our main results.

\footnotetext{
${ }^{1}$ Research supported in part by National Science Foundation.
} 
Theorem 1. Let $M^{n}$ be a compact $n$-dimensional manifold with a symplectic or volume form $\omega$. Let $\phi: M^{n} \rightarrow M^{n}$ be a diffeomorphism of class $C^{1}$ that preserves $\omega$. In other words, $\phi \in \operatorname{Diff}_{\omega}^{1}\left(M^{n}\right)$. Let $p \in M^{n}$ be a hyperbolic periodic point of $\phi$. Then for any given $q \in W_{\phi}^{u}(p)$ (or $W_{\phi}^{u}(p)$ ) and neighborhoods $U$ of $q$ in $M^{n}$ and $V$ of $\phi$ in $\operatorname{Diff}_{\omega}^{1}\left(M^{n}\right)$, there is a $\phi^{\prime} \in V$ such that:

1. $p$ is a hyperbolic periodic point of $\phi^{\prime}$,

2. $p$ has a homoclinic point with respect to $\phi^{\prime}$ in $U$.

Recall that a subset $\mathscr{B} \subset \operatorname{Diff}_{\omega}^{r}\left(M^{n}\right)$ is residual if it contains a countable intersection of open dense sets. Residual sets are dense and a countable intersection of residual sets is again residual. Properties which are true for a residual set are called generic.

According to Robinson's theorems [9], the homoclinic point we obtained above can be assumed to be transversal. Since transversal homoclinic points persist under small perturbations, a standard argument yields the following results:

Theorem 2. Let $M^{n}$ be a compact $n$-dimensional manifold with a symplectic or volume form $\omega$. Then there is a residual subset $\mathscr{B} \subset \operatorname{Diff}_{\omega}^{1}\left(M^{n}\right)$ such that if $\phi \in \mathscr{B}$ and $p \in M^{n}$ are such that $p$ is a hyperbolic periodic point of $\phi$, then $W_{\phi}^{s}(p) \cap$ $W_{\phi}^{u}(p)$ is dense in both $W_{\phi}^{s}(p)$ and $W_{\phi}^{u}(p)$.

The homoclinic point has played an important role in dynamical system and Hamiltonian dynamics and it goes back to the last century. Poincaré discovered the homoclinic phenomenon and its associated chaotic dynamics in his study of the restricted three body problem in celestial mechanics [5]. Poincare suspected that transverse homoclinic points occurred generically in the Hamiltonian systems and conjectured that they would be everywhere dense in both stable and unstable manifolds. Our result positively answers Poincaré's question in $C^{1}$ topology on any compact manifold.

Our results above are generalizations of Takens's results for compact surfaces [11]. Takens showed that if $M^{n}$ is a compact surface $(n=2)$, then generically transverse homoclinic points are dense in both stable and unstable manifolds, in $C^{1}$ topology. For manifolds with dimension larger than two, Takens's method gives the generic existence of a transverse homoclinic point for any hyperbolic periodic point. However, his proof fails to produce the density result.

Unlike Takens's proof for the compact surfaces, the proof of our main theorems uses the volume-preserving property very little and thus can be applied to more general cases. In fact, our proof is very much similar in flavor to a connecting lemma recently announced by Hayashi [1]. In fact, we borrowed Hayashi's idea of cutting pieces of an orbit so that the resulting connecting orbit can bypass the bad portions of the orbit. Since the details of Hayashi's work is not available to us, here we present a different and easier construction and we apply it to symplectic and volume preserving diffeomorphisms.

The basic idea is to improve the closing lemma (Pugh [6], Pugh \& Robinson [8]) So that one can connect the stable manifold and the unstable manifold to obtain homoclinic or heteroclinic points. The basic difficulty in connecting or closing orbits for a $C^{1}$ diffeomorphism is the intervention of intermediate points in the orbits. Here we adopt a selection process that successively eliminates all the interventions. Combining our selection process with Pugh's closing lemma (especially the part on sequence of linear isomorphisms of $\mathbb{R}^{n}$ ), one can obtain various types of the $C^{1}$ 
connecting lemma. In particular, it positively answers almost all questions raised by Pugh [7] on compact manifolds. As a simple example, we state the following simple connecting lemma.

Theorem 3. Let $M^{n}$ be an $n$-dimensional manifold (not necessarily compact). Let $\phi: M^{n} \rightarrow M^{n}$ be a $C^{1}$ diffeomorphism of $M^{n}$. Let $p \in M^{n}$ be a hyperbolic periodic point of $\phi$. If

or

$$
q \in \operatorname{closure}\left(W_{\phi}^{s}(p)\right) \cap W_{\phi}^{u}(p) \backslash\{p\}
$$

$$
q \in \operatorname{closure}\left(W_{\phi}^{u}(p)\right) \cap W_{\phi}^{s}(p) \backslash\{p\},
$$

then for any $C^{1}$ neighborhood $U$ of $\phi$ in $\operatorname{Diff}^{1}\left(M^{n}\right)$ and a neighborhood $V$ of $q$ in $M^{n}$, there exists $\psi \in U$ such that the orbit of $p$ under $\phi$ is not in the support of $(\psi-\phi)$ (thus $p$ is a hyperbolic periodic point for $\psi)$, and $p$ has a homoclinic point in $V$.

This is one of the basic results announced by Hayashi [1].

To end this section, we would like to mention two related results on generic existence of homoclinic points in $C^{r}$ topology. Using an idea from Robinson [10], Pixton [4] showed that $C^{r}$ generically for any $r \geqq 1$, any hyperbolic periodic point on $S^{2}$ has a homoclinic point. Oliveira [3] extended Pixton's result to the two-torus $T^{2}$, again for $C^{r}$ topology. So far there are no density results in $C^{r}$ topology.

\section{A Perturbation Lemma and Recurrent Points}

In this section, we prove a known perturbation lemma (cf. $[2,9,11]$ ).

Let $d$ be a metric on $M^{n}$ induced from some Riemann structure and let $B_{\delta}(x)$ denote the set of $y \in M^{n}$ with $d(x, y)<\delta$. We also let $\bar{B}_{\delta}(x)$ denote the closure of $B_{\delta}(x)$.

Lemma 1 (Perturbation Lemma). Let $M^{n}$ be an $n$-dimensional compact manifold. Fix $\phi \in \operatorname{Diff}_{\omega}^{r}\left(M^{n}\right), r \geqq 1$. There exist constants $\varepsilon_{0}>0$ and $c>0$, depending on $\phi$, such that for any $x \in M^{n}$, and any $\psi \in \operatorname{Diff}_{\omega}^{r}\left(M^{n}\right)$ such that $\|\phi-\psi\|_{C^{r}}<\varepsilon_{0}$, and any positive numbers $0<\delta \leqq \varepsilon_{0}, 0<\varepsilon \leqq \varepsilon_{0}$, the following facts hold:

if $d(y, x)<c \delta^{r} \varepsilon$, then there is a $\psi_{1} \in \operatorname{Diff}_{\omega}^{r}\left(M^{n}\right),\left\|\psi_{1}-\psi\right\|_{C^{r}}<\varepsilon$ such that $\psi_{1} \psi^{-1}(x)=y, \psi_{1}(z)=\psi(z)$ for all $z \notin \psi^{-1}\left(B_{\delta}(x)\right)$, and $\psi^{-1}(z)=\psi_{1}^{-1}(z)$ for all $z \notin B_{\delta}(x)$.

Proof. We first introduce the generating functions. A generating function provides a very convenient tool in the study of symplectic diffeomorphisms.

Let $(u, v)=\left(u_{1}, \ldots, u_{k}, v_{1}, \ldots, v_{k}\right)$ be coordinates on $\mathbb{R}^{2 k}, \omega=\sum_{i=1}^{n} d u_{i} \wedge d v_{i}$. Let $f(u, v)=(\xi(u, v), \eta(u, v))$ be a $C^{r}$ diffeomorphism, preserving $\omega$, defined on a simply connected neighborhood $V$ of the origin. Thus $\sum_{i=1}^{k} d u_{i} \wedge d v_{i}=$ $\sum_{i=1}^{k} d \xi_{i} \wedge d \eta_{i}$. Let $\left(\xi^{0}, \eta^{0}\right)=f(0,0)$, we may assume that $\frac{\partial \eta}{\partial v}(u, v)$ is non-singular at each point of $V$. This implies that we can solve from $\eta=\eta(u, v)$ to obtain $v=v(u, \eta)$ and thus $\left(u_{1}, \ldots, u_{k}, \eta_{1}, \ldots, \eta_{k}\right)$ defines new $C^{r}$ coordinates on a small neighborhood of $\left(0, \eta^{0}\right)$. Since $f$ is symplectic, the 1 -form $\alpha=\sum_{i=1}^{k}\left(\xi_{i} d \eta_{i}+v_{i} d u_{i}\right)$ is closed, so there is a $C^{r+1}$ real valued function $S=S(u, \eta)$, unique up to a constant, defined for $(u, \eta)$ near $\left(0, \eta^{0}\right)$ such that $d S=\alpha=\sum_{i=1}^{k}\left(\xi_{i} d \eta_{i}+v_{i} d u_{l}\right) . S$ is 
called a generating function and it satisfies $\frac{\partial S}{\partial \eta_{i}}=\xi_{i}, \frac{\partial S}{\partial u_{i}}=v_{i}$ and $\frac{\partial^{2} S}{\partial u \partial \eta}$ is nonsingular for each $(u, \eta)$ near $\left(0, \eta^{0}\right)$ in the domain of $S$.

Conversely, let $S(u, \eta)$ be a $C^{r+1}$ function, defined for $(u, \eta)$ in a small neighborhood of $\left(0, \eta^{0}\right)$, such that $\frac{\partial^{2} S}{\partial u \partial \eta}$ is non-singular for each $(u, \eta)$, then setting $\xi_{i}(u, \eta)=\frac{\partial S}{\partial \eta_{i}}(u, \eta)$ and $v_{i}(u, \eta)=\frac{\partial S}{\partial u_{i}}(u, \eta)$, we may solve for $\eta$ in terms of $(u, v)$ and obtain a symplectic diffeomorphism $(u, v) \rightarrow(\xi(u, v), \eta(u, v))$.

We proceed to prove the perturbation lemma. Since all the perturbations we do are local, we will use local coordinates.

Let $\lambda: \mathbb{R} \rightarrow[0,1]$ be a $C^{\infty}$ real function so that $\lambda(z)=1$ for $z \leqq 1 / 2$ and $\lambda(z)=0$ for $z \geqq 1$, and let $\beta=\sup _{z \in \mathbb{R}}\left\{1,\left|\lambda^{\prime}(z)\right|, \ldots,\left|\lambda^{(r+1)}(z)\right|\right\}$. The function $S_{1}(u, \eta)=\left(u_{1}+\mu\right) \eta_{1}+\sum_{i=2}^{k} u_{i} \eta_{i}$ is a generating function for the translation $\left(u_{1}, \ldots, u_{k}, v_{1}, \ldots, v_{k}\right) \rightarrow\left(u_{1}+\mu, u_{2}, \ldots, u_{k}, v_{1}, \ldots, v_{k}\right)$, while the function $S_{0}(u, \eta)=$ $\sum_{i=1}^{k} u_{i} \eta_{i}$ is a generating function for the identity map id.

For $\delta>0$ small, let

$$
S(u, \eta)=\lambda\left(\frac{|(u, \eta)|}{\delta}\right) S_{1}(u, \eta)+\left[1-\lambda\left(\frac{|(u, \eta)|}{\delta}\right)\right] S_{0}(u, \eta) .
$$

Then $S(u, \eta)$ is a $C^{\infty}$ function and $S(u, \eta)=S_{1}(u, \eta)$ for $|(u, \eta)| \leqq \delta / 2$, and $S(u, \eta)$ $=S_{0}(u, \eta)$ for $|(u, \eta)| \geqq \delta$. A direct computation shows that $\left\|S-\bar{S}_{0}\right\|_{r+1} \leqq \beta \bar{c} \mu \delta^{-r}$, where $\bar{c}$ is a constant depending only on $k$ and $r$.

Let $h^{*}(u, \eta)$ be a diffeomorphism of $\mathbb{R}^{2 k}$ defined by $h^{*}(u, \eta)=\left(\frac{\partial S}{\partial u}(u, \eta)\right.$, $\left.\frac{\partial S}{\partial \eta}(u, \eta)\right)$, then we have $\left\|h^{*}-\mathrm{id}\right\|_{C^{r}} \leqq \beta \bar{c} \mu \delta^{-r}$. For $\mu$ small, one can solve for $\eta$ from $v_{i}=\frac{\partial S}{\partial u_{i}}(u, \eta)$ to obtain $\eta=\eta(u, v)$. Let $h(u, v)$ be the symplectic diffeomorphism of $\mathbb{R}^{2 k}$ defined by $h(u, v)=h^{*}(u, \eta(u, v))$. Then there exists a constant $\tilde{c}$ such that $\|h-\mathrm{id}\|_{C^{r}} \leqq \beta \bar{c} \tilde{c} \mu \delta^{-r}$. Note that if $\mu<\varepsilon(\beta \bar{c} \tilde{c})^{-1} \delta^{r}$ for any $\varepsilon>0$, then $\|h-\mathrm{id}\|_{C^{r}}<\varepsilon$.

To summarize, we have proved the following fact: For any compact manifold, there are $\delta_{0}>0$ and $c_{0}>0$ such that for any point $x \in M^{n}$, any $0<\delta \leqq \delta_{0}$ and any $y \in M^{n}$ with $d(x, y) \leqq c_{0} \delta^{r} \varepsilon$, then there is a symplectic diffeomorphism $h \in \operatorname{Diff}_{\omega}^{r}\left(M^{n}\right)$ with support $(h) \subset B_{\delta}(x)$ and $\|h-\mathrm{id}\|_{C^{r}}<\varepsilon$ such that $h(x)=y$.

To finish the proof of the lemma, we just need to compose a given map $\psi$ with $h$ to obtain $\psi_{1}=h \psi$. To obtain $\left\|\psi-\psi_{1}\right\|_{C^{r}}<\varepsilon$ for all $\psi$, we need $\|\psi-\phi\|_{C^{r}} \leqq \varepsilon_{0}$ for some $\varepsilon_{0}>0$. Since $h$ is also volume preserving, the proof works for volumepreserving diffeomorphisms when the dimension $M^{n}$ is even. When $n$ is odd, we take the identity map in one direction perpendicular to $y-x$ (in local coordinates) and use $h$ in the remaining directions.

This proves the lemma.

Now we use the perturbation lemma to prove the following result:

Lemma 2. Let $\phi \in \operatorname{Diff}_{\omega}^{r}\left(M^{n}\right)$ and let $p \in M^{n}$ be a hyperbolic periodic point of period $k$, with respect to $\phi$. For any $\varepsilon>0$, any $q \in W_{\phi}^{u}(p)$ and any neighborhood $U$ of $q$, there exist a $\phi^{\prime} \in \operatorname{Diff}_{\omega}^{r}\left(M^{n}\right),\left\|\phi-\phi^{\prime}\right\|_{C^{r}}<\varepsilon$ such that

1. Support $\left(\phi-\phi^{\prime}\right) \subset U$ and hence, $p$ is a hyperbolic periodic point of period $k$ for $\phi^{\prime}$,

2. $q \in W_{\phi^{\prime}}^{u}(p)$,

3. $q$ is a recurrent point under $\phi^{\prime}$. 
Recall that a point $q \in M^{n}$ is a recurrent point under $\phi^{\prime}$ if there exists a sequence of positive integers $\left\{n_{i}\right\}_{i=1}^{\infty}, n_{i} \rightarrow \infty$ as $i \rightarrow \infty$ such that $\left(\phi^{\prime}\right)^{n_{i}}(q) \rightarrow q$ as $i \rightarrow \infty$.

Proof. Let $\varepsilon>0$ be given. For any $q \in W_{\phi}^{u}(p)$ and $U \subset M^{n}$, a small neighborhood of $q$, choose $\delta_{1}$ small so that $B_{\delta_{1}}(q) \subset U$. Consider the small ball $B^{1}=B_{c \delta_{1}^{r} \varepsilon_{1}}(q)$, where $c$ is given by the perturbation lemma and $0<\varepsilon_{1} \leqq \varepsilon / 2$. Since $\phi$ preserves the volume and $M^{n}$ is compact, there exists a positive integer $j_{1}$ such that $\phi^{j_{1}}\left(B^{1}\right) \cap B_{\delta_{1}}(q) \neq \emptyset$ and $\phi^{i}\left(B^{1}\right) \cap B_{\delta_{1}}(q)=\emptyset$ for all $i=1,2, \ldots, j_{1}-1$. This implies that there exists a point $q_{1} \in B_{\delta_{1}}(q)$ such that $\phi^{j_{1}}\left(q_{1}\right) \in B_{\delta_{1}}(q)$ and $\phi^{i}\left(q_{1}\right) \notin B_{\delta_{1}}(q)$, for all $i=1,2, \ldots, j_{1}-1$. Now we apply the perturbation lemma to obtain $\phi_{1} \in \operatorname{Diff}_{\omega}^{r}\left(M^{n}\right)$ with $\left\|\phi_{1}-\phi\right\|_{C^{r}}<\varepsilon_{1} \leqq \varepsilon / 2$ and $\operatorname{Support}\left(\phi-\phi_{1}\right) \subset$ $B_{\delta_{1}}(q)$ such that $\phi_{1}(q)=\phi\left(q_{1}\right)$. Thus $\left(\phi_{1}\right)^{j_{1}}(q) \in B_{\delta_{1}}(q)$.

Now, since $\left(\phi_{1}\right)^{j_{1}}(q) \neq q$, we may choose $0<\delta_{2}<\delta_{1} / 2$ so that $\left(\phi_{1}\right)^{j}(q) \notin$ $B_{\delta_{2}}(q)$. Let $B^{2}=B_{c \delta_{2} \varepsilon_{2}}(q)$, where $0<\varepsilon_{2} \leqq \varepsilon / 4$. We choose $\delta_{2}$ and $\varepsilon_{2}$ so small such that for all $\phi^{\prime} \in \operatorname{Diff}_{\omega}^{r}\left(M^{n}\right)$, with $\left\|\phi^{\prime}-\phi\right\|_{C^{r}} \leqq \varepsilon_{2}$, and any point $x \in B^{2}$, we have $\left(\phi^{\prime}\right)^{j_{1}}(x) \in B_{\delta_{1}}(q) \backslash B_{\delta_{2}}(q)$ and $\left(\phi^{\prime}\right)^{i}(x) \notin B_{\delta_{1}}(q)$ for all $i=1,2, \ldots, j_{1}-1$.

With the same argument, we see that there is a point $q_{2} \in B^{2}$ and an integer $j_{2}>j_{1}$ such that $\left(\phi_{1}\right)^{j_{2}}\left(q_{2}\right) \in B_{\delta_{2}}(q)$ and $\left(\phi_{1}\right)^{i}\left(q_{2}\right) \notin B_{\delta_{2}}(q)$, for all $i=1,2, \ldots, j_{2}-1$. Again we apply the perturbation lemma to obtain $\phi_{2} \in$ $\operatorname{Diff}_{\omega}^{r}\left(M^{n}\right)$ with $\left\|\phi_{1}-\phi_{2}\right\|_{C^{r}}<\varepsilon_{2} \leqq \varepsilon / 4$ and $\operatorname{Support}\left(\phi_{2}-\phi_{1}\right) \in B_{\delta_{2}}(q)$ such that $\phi_{2}(q)=\phi_{1}\left(q_{2}\right)$. Observe that $\left(\phi_{2}\right)^{j_{1}}(q) \in B_{\delta_{1}}(q)$ and $\left(\phi_{2}\right)^{j_{2}}(q) \in B_{\delta_{2}}(q)$.

Continue the above process, we obtain a sequence of real positive numbers $\delta_{1}, \delta_{2}, \ldots$, a sequence of integers $0<j_{1}<j_{2}<\ldots$, and a sequence of functions $\phi_{1}, \phi_{2}, \ldots, \in \operatorname{Diff}_{\omega}^{r}\left(M^{n}\right)$ such that $\left(\phi_{i}\right)^{j_{k}} \in B_{\delta_{k}}(q)$ for all $k=1,2, \ldots, i$.

Let $\phi^{\prime}=\lim _{i \rightarrow \infty} \phi_{i} \in \operatorname{Diff}_{\omega}^{r}\left(M^{n}\right)$, then $\left\|\phi^{\prime}-\phi\right\|_{C^{r}}<\varepsilon$ and $q$ is a recurrent point of $\phi^{\prime}$.

This proves the lemma.

\section{Boxes and Sequence of Perturbations}

In this section, we are concerned with linear maps and their perturbations on $\mathbb{R}^{n}$. Let $\omega$ be the standard symplectic form or volume form on $\mathbb{R}^{n}$ and let $f \in \operatorname{Diff}_{\omega}^{1}\left(\mathbb{R}^{n}\right)$ be a linear map of the form

$$
f\left(x_{1}, x_{2}, \ldots, x_{n}\right)=\left(\lambda_{1} x_{1}, \lambda_{2} x_{2}, \ldots, \lambda_{n} x_{n}\right)
$$

with all $\lambda_{i}$ 's real, $\left|\lambda_{i}\right| \neq 1, i=1,2, \ldots, n$.

For any set of positive numbers $a_{1}>0, a_{2}>0, \ldots, a_{n}>0$, let $\mathbf{x}=\left(x_{1}\right.$, $\left.x_{2}, \ldots, x_{n}\right) \in \mathbb{R}^{n}$, we define the following rectangular box with size $\left(a_{1}, a_{2}, \ldots, a_{n}\right)$ :

$$
D_{\mathbf{x}}\left(a_{1}, a_{2}, \ldots, a_{n}\right)=\left\{\left(y_{1}, y_{2}, \ldots, y_{n}\right) \in \mathbb{R}^{n}|| x_{i}-y_{i} \mid \leqq a_{i}\right\},
$$

and for any $\alpha>0$, we define

$$
\alpha D_{\mathbf{x}}\left(a_{1}, a_{2}, \ldots, a_{n}\right)=\left\{\left(y_{1}, y_{2}, \ldots, y_{n}\right) \in \mathbb{R}^{n}|| x_{i}-y_{i} \mid \leqq \alpha a_{i}\right\} .
$$

We have the following lemma. 
Lemma 3. Let $\varepsilon>0, R>0, \tau \geqq 1$ and $0<\alpha<1$ be given ( $\varepsilon$ and $(1-\alpha)$ will be in general very small). Then there exist an integer $N$ and an $n$-tuple $\left(a_{1}, a_{2}, \ldots, a_{n}\right)$ of positive numbers, depending only on $\lambda_{1}, \lambda_{2}, \ldots, \lambda_{n}, \varepsilon, \tau$ and $\alpha$, such that for any rectangular box $D_{\mathbf{x}^{*}}\left(\xi_{1}, \xi_{2}, \ldots, \xi_{n}\right)$ with

$$
\left(\xi_{i} / a_{i}\right) /\left(\xi_{j} / a_{j}\right) \leqq \tau
$$

for all $i, j=1,2, \ldots, n$;

$$
\bigcup_{i=0}^{N-1} f^{i}\left(D_{\mathbf{x}^{*}}\left(\xi_{1}, \xi_{2}, \ldots, \xi_{n}\right)\right) \subset B_{R}(O)
$$

and

$$
D_{\mathbf{x}^{*}}\left(\xi_{1}, \xi_{2}, \ldots, \xi_{n}\right) \cap f\left(D_{\mathbf{x}^{*}}\left(\xi_{1}, \xi_{2}, \ldots, \xi_{n}\right)\right)=\emptyset,
$$

and any two points $x^{1}, x^{2} \in \alpha D_{\mathbf{x}^{*}}\left(\xi_{1}, \xi_{2}, \ldots, \xi_{n}\right)$, there is a $g \in \operatorname{Diff}_{\omega}^{1}\left(\mathbb{R}^{n}\right)$ such that

1. $\|f-g\|_{C^{1}}<\varepsilon$,

2. $\operatorname{support}(f-g) \subset \bigcup_{i=0}^{N-1} f^{i}\left(D_{\mathbf{x}^{*}}\left(\xi_{1}, \xi_{2}, \ldots, \xi_{n}\right)\right)$,

3. $g^{N}\left(x^{2}\right)=f^{N}\left(x^{1}\right)$.

Proof. Without loss of generality, we assume that

$$
\left|\lambda_{1}\right|>\left|\lambda_{2}\right|>\cdots>\left|\lambda_{n}\right|
$$

We need some estimates on the distance from $f^{i}\left(\alpha D_{\mathbf{x}^{*}}\left(\xi_{1}, \xi_{2}, \ldots, \xi_{n}\right)\right)$ to the boundary of $f^{i}\left(D_{\mathbf{x}^{*}}\left(\xi_{1}, \xi_{2}, \ldots, \xi_{n}\right)\right)$. It is easy to see that

$$
f^{i}\left(D_{\mathbf{x}^{*}}\left(\xi_{1}, \xi_{2}, \ldots, \xi_{n}\right)\right)=D_{f^{i}\left(\mathbf{x}^{*}\right)}\left(\left|\lambda_{1}\right|^{i} \xi_{1},\left|\lambda_{2}\right|^{i} \xi_{2}, \ldots,\left|\lambda_{n}\right|^{i} \xi_{n}\right) .
$$

If $\left|\lambda_{j}\right|^{i} \xi_{j} \leqq\left|\lambda_{l}\right|^{i} \xi_{l}$ for all $l=1,2, \ldots, n$, then

$$
d\left(x, f^{i}\left(D_{\mathbf{x}^{*}}\left(\xi_{1}, \xi_{2}, \ldots, \xi_{n}\right)\right) \geqq(1-\alpha)\left|\lambda_{j}\right|^{i} \xi_{j}\right.
$$

for all $x \in f^{i}\left(\alpha D_{\mathbf{x}^{*}}\left(\xi_{1}, \xi_{2}, \ldots, \xi_{n}\right)\right)$.

Let $x^{1}=\left(x_{1}^{1}, x_{2}^{1}, \ldots, x_{n}^{1}\right)$ and $x^{2}=\left(x_{1}^{2}, x_{2}^{2}, \ldots, x_{n}^{2}\right)$. We divide our perturbation process into $n$ steps. In the first step, which takes $N_{1}$ iterates of $f$, we do the perturbations that push $x^{2}$ towards $x^{1}$ in $x_{1}$ direction (i.e., in the direction of $\left.e_{1}=(1,0, \ldots, 0)\right)$. In the second step, we perturb $f$ to push $x^{2}$ towards $x^{1}$ in $x_{2}$ direction. The second step takes $N_{2}-N_{1}$ iterates of $f$. Then the third step takes $N_{3}-N_{2}$ and we push $x^{2}$ in $x_{3}$ direction and so on. The total number of steps we need is $N=N_{n}$, which, as we shall see later, depends only on $\varepsilon, \alpha, \tau$ and $\lambda_{1}, \lambda_{2}, \ldots, \lambda_{n}$.

However, due to the distorsion of the rectangular $D_{\mathbf{x}^{*}}\left(\xi_{1}, \xi_{2}, \ldots, \xi_{n}\right)$ from the standard $D_{\mathbf{x}^{*}}\left(a_{1}, a_{2}, \ldots, a_{n}\right)$, in between any two steps, we also need some transitional iterates $N_{t}$ without doing any pushes. The transition is needed to correct the distorsion.

We now begin to choose the $n$-tuple $\left(a_{1}, a_{2}, \ldots, a_{n}\right)$. In order to reach optimal effect for each perturbation, the choice of $\left(a_{1}, a_{2}, \ldots, a_{n}\right)$ is so that when we do perturbations in the $x_{i}$ direction, under the $j^{\text {th }}$ iterate of $f$, we require that the shortest distance between the set $f^{j}(\alpha D)$ and the boundary of $f^{j}(D)$ is reached in the $x_{i}$ direction, where $D=D_{\mathbf{x}^{*}}\left(\xi_{1}, \xi_{2}, \ldots, \xi_{n}\right)$. In other words, we need the following *-condition:

$$
*\left|\lambda_{i}\right|^{j} \xi_{i} \leqq\left|\lambda_{l}\right|^{j} \xi_{l} \text { for all } N_{i-1}+N_{t} \leqq j<N_{i} \text { and } l=1,2, \ldots, n .
$$


First, we calculate the number of pushes we need in each direction. For a moment, let's assume that the *-condition is satisfied. Then the number of perturbations needed to push a point from one side of the inner box to the opposite side along the $x_{i}$ direction, by $\varepsilon$ small $C_{1}$ perturbations, is $\frac{\alpha}{(1-\alpha) c \varepsilon}$, where $c$ is given by the perturbation lemma. We point out that the perturbation lemma can be applied to the compact subset the closure of $B_{R}(O) \subset \mathbb{R}^{n}$.

We fix a positive integer

$$
N_{1}>\frac{\alpha}{(1-\alpha) c \varepsilon} \text {. }
$$

Now choose $a_{1}>0$ small so that $\bigcup_{i=0}^{N} f^{i}(D) \subset B_{M}(O)$. As the first step, we need to find $f_{1} \in \operatorname{diff}_{\omega}^{1}\left(\mathbb{R}^{n}\right)$ such that $f_{1}^{N_{1}}\left(x_{1}^{2}, x_{2}^{2}, \ldots, x_{n}^{2}\right)=f^{N_{1}}\left(x_{1}^{1}, x_{2}^{2}, \ldots, x_{n}^{2}\right)$. For this, it suffices to have $a_{i} \geqq\left|\lambda_{1} / \lambda_{i}\right|^{N_{1}} a_{1}$ for all $2 \leqq i \leqq n$. This implies the $*$-condition for the first $N_{1}$ iterates of $f$.

Let $N_{t}$ be a positive integer such that

$$
\left|\lambda_{i} / \lambda_{i+1}\right|^{N_{t}}>\tau \text { for all } i=1, \ldots, n-1 .
$$

We now choose $a_{2}$ such that

$$
\left|\lambda_{1} / \lambda_{2}\right|^{N_{1}+N_{t}-1} a_{1} \leqq a_{2} \leqq\left|\lambda_{1} / \lambda_{2}\right|^{N_{1}+N_{t}} a_{1}
$$

Now, starting from the $N_{1}+N_{t}^{\text {th }}$ iterate of $f_{1}$, we do another $N_{1}$ pushes and this time in the $x_{2}$ direction. We find an $f_{2} \in \operatorname{diff}_{\omega}^{1}\left(\mathbb{R}^{n}\right)$ such that $f_{2}^{N_{2}}\left(x_{1}^{2}, x_{2}^{2}, \ldots, x_{n}^{2}\right)=$ $f^{N_{2}}\left(x_{1}^{1}, x_{2}^{1}, x_{3}^{2}, \ldots, x_{n}^{2}\right)$, where $N_{2}=N_{1}+N_{t}+N_{1}$. In this step, we need $a_{i} \geqq$ $\left|\lambda_{2} / \lambda_{i}\right|^{N_{2}} a_{2}$ for all $3 \leqq i \leqq n$.

We repeat the above process. Suppose that $a_{1}, a_{2}, \ldots, a_{i}, i<n$, are chosen. Let $N_{i}=N_{i-1}+N_{t}+N_{1}$, we then choose $a_{i+1}$ to be a positive real number such that

$$
\left|\lambda_{i} / \lambda_{i+1}\right|^{N_{i}+N_{t}-1} a_{i} \leqq a_{i+1} \leqq\left|\lambda_{i} / \lambda_{i+1}\right|^{N_{i}+N_{t}} a_{i}
$$

For $n$-tuple positive real numbers $\left(a_{1}, a_{2}, \ldots, a_{n}\right)$ thus chosen, we see that

$$
\left|\lambda_{i}\right|^{j} a_{i}\left|\frac{\lambda_{i}}{\lambda_{i+1}}\right|^{N_{t}} \leqq\left|\lambda_{l}\right|^{j} a_{l}
$$

for all $N_{i-1}+N_{t} \leqq j<N_{i}$ and for all $l \neq i$. This implies that the $*$-condition is satisfied for the $n$-tuple $\left(a_{1}, a_{2}, \ldots, a_{n}\right)$ and we can carry out the perturbation procedure. The lemma is proved for the boxes $D_{\mathbf{x}^{*}}\left(a_{1}, a_{2}, \ldots, a_{n}\right)$.

To complete the proof of our lemma, we only need to note that for any $\left(\xi_{1}, \xi_{2}, \ldots, \xi_{n}\right)$, with $\left(a_{i} / \xi_{i}\right) /\left(a_{j} / \xi_{j}\right) \leqq \tau$, by the selection of $\left(a_{1}, a_{2}, \ldots, a_{n}\right)$ and the choice of $N_{t}$, the $*$-condition is satisfied.

This proves the lemma.

We remark that in the proof of the above lemma, one can relax the $*$-condition a little and replace it by

$$
\left|\lambda_{i}\right|^{j} \xi_{i} \leqq \tau\left|\lambda_{l}\right|^{j} \xi_{l} \text { for all } N_{i-1}+N_{t} \leqq j<N_{i} \text { and } l=1,2, \ldots, n .
$$

In this way, one needs to increase $N_{i}$ by making smaller pushes in each step. The advantage is that one does not need the intermediate steps $N_{t}$. This is useful when one has multiple or complex eigenvalues for a linear map $f$. 
The above lemma can be generalized to non-linear maps in a small neighborhood of hyperbolic fixed points. According to Robinson [9], for a generic diffeomorphism, all fixed points have simple eigenvalues.

Lemma 4. Let $f: \mathbb{R}^{n} \rightarrow \mathbb{R}^{n}$ be a symplectic or volume-preserving diffeomorphism of class $C^{1}$. Suppose that the origin $O$ is a hyperbolic fixed point of $f$ with all eigenvalues simple. Further assume that $f$ is of the form $f(\mathbf{x})=$ $A \mathbf{x}+o(\|\mathbf{x}\|)$, where the matrix $A$ is in Jordan canonical form. Then for any $\varepsilon>0, \tau>0$ and $0<\alpha<1$, there exists $\delta>0$ such that if restricted to the domain $U=\left\{\mathbf{x} \in \mathbb{R}^{n} \mid\|\mathbf{x}\|<\delta\right\}$, i.e., if $\bigcup_{i=0}^{N} f^{i}\left(D_{\mathbf{x}^{*}}\left(\xi_{1}, \xi_{2}, \ldots, \xi_{n}\right)\right) \subset U$, then the conclusions of Lemma 3 remain true.

Proof. The proof of this lemma basically follows from the proof of Lemma 3. Since $N$ is a pre-determined number, the effect of the higher order term $o(\|\mathbf{x}\|)$, within $N$ iterates of $f$, can be safely neglected. The only modification we need is to consider the case with complex eigenvalues. It turns out that one just needs to consider each of the complex pairs simultaneously in the perturbation sequence. The proof is similar to the case with all eigenvalues real. We omit the details.

Another way to obtain a lemma similar to Lemma 4 is to do a small perturbation so that $f$ is a linear function in a small neighborhood $B_{\delta}(O)$ of the origin and $f$ remains the same outside of $B_{2 \delta}(O)$. In order for the perturbation to be symplectic, one may use the generating functions in the perturbation. The volume preserving case can be dealt in a similar way.

\section{A Selection Procedure}

In this section, we introduce a selection process that chooses appropriate points for the final perturbation. The idea is to successively eliminate all the intermediate interventions so that one can join two end points of a finite segment of an orbit, by a small $C^{1}$ perturbation.

Let $x^{0}, x^{1}, \ldots, x^{m}$ be a finite sequence of points on $M^{n}$, for some positive integer $m$. Since our discussions of this section will be restricted to a small neighborhood of $x^{0}$, without loss of generality, we may assume that our manifold is $\mathbb{R}^{n}$. Let $d$ be the standard Euclidean metric on $\mathbb{R}^{n}$, we assume that $d\left(x^{i}, x^{0}\right)>d\left(x^{1}, x^{0}\right)$, for all $i>1$. In other words, $x^{1}$ is the closest point to $x^{0}$ in the sequence.

Let $\alpha_{1}, 1 / 2<\alpha_{1}<1$, be a positive number depending only on the dimension of $M^{n}$ such that

$$
\sqrt{\frac{4 n-1}{4 n}}<\alpha_{1}<1 .
$$

$\alpha_{1}$ will be fixed throughout the paper.

Let $x^{0}=\left(x_{1}^{0}, x_{2}^{0}, \ldots, x_{n}^{0}\right)$ and $x^{1}=\left(x_{1}^{1}, x_{2}^{1}, \ldots, x_{n}^{1}\right)$. Let $i^{*}, 1 \leqq i^{*} \leqq n$ be an integer such that $\left|x_{i^{*}}^{0}-x_{i^{*}}^{1}\right| \geqq\left|x_{i}^{0}-x_{i}^{1}\right|$ for all $i=1,2, \ldots, n$. Obviously $\left|x_{i^{*}}^{0}-x_{i^{*}}^{1}\right| \geqq$ $d\left(x^{0}, x^{1}\right) / \sqrt{n}$.

Let $\bar{x}^{0}=\left(x^{0}+x^{1}\right) / 2$ and let

$$
\bar{x}_{ \pm}^{0}=\bar{x}^{0} \pm \frac{\left(x_{i^{*}}^{1}-x_{i^{*}}^{0}\right)}{4} \mathbf{e}_{i^{*}}
$$


where $\mathbf{e}_{i *}$ is the $i^{\text {*th }}$ unit coordinate vector. A simple calculation shows that $d\left(x^{0}, \bar{x}_{-}^{0}\right)<\alpha_{1} d\left(x^{0}, x^{1}\right)$ and $d\left(x^{1}, \bar{x}_{+}^{0}\right)<\alpha_{1} d\left(x^{0}, x^{1}\right)$. Moreover, the two balls centered at $\bar{x}_{ \pm}^{0}$ with radius $\alpha_{1} d\left(x^{0}, x^{1}\right) / 2$ cover a rectangular region (with all sides parallel to coordinate planes) containing $x^{0}$ and $x^{1}$. This fact will be used later.

We have the following lemma.

Lemma 5 (Selection Lemma). Let $\left\{x^{i}\right\}_{i=0}^{m}$ be a finite sequence such that $d\left(x^{i}, x^{0}\right)>$ $d\left(x^{1}, x^{0}\right)$ for all $i>1$. Then there exists a finite subsequence $\left\{x^{n_{i}}\right\}_{i=0}^{2 j^{*}+1}$, for some positive integer $j^{*}$ such that

1. $n_{0}=0$ and $n_{1}=1$, i.e., $x^{0}$ and $x^{1}$ are the first two points in subsequence.

2. $d\left(x^{0}, x^{n_{1}}\right) \leqq \frac{d\left(x^{0}, x^{1}\right)}{1-\alpha_{1}}$, where $\alpha_{1}$ is the constant defined above.

3. For each pair $x^{n_{2 j}}, x^{n_{2 j+1}}$, define $\bar{x}^{i}, \bar{x}_{+}^{i}$ and $\bar{x}_{-}^{i}$ in the same way as $\bar{x}^{0}, \bar{x}_{+}^{0}$ and $\bar{x}_{-}^{0}$ are defined above. If $n_{2 j+1}<i<n_{2 j+2}$ for some $j=0,1, \ldots, j^{*}$, then

$$
x^{i} \notin \bigcup_{j=0}^{j^{*}}\left(\bar{B}_{\alpha_{1} d\left(x^{n_{2 j} j}, x^{\left.n_{2 j+1}\right) / 2}\right.}\left(\bar{x}_{+}^{j}\right) \cup \bar{B}_{\alpha_{1} d\left(x^{n_{2 j}}, x^{\left.n_{2 j+1}\right) / 2}\right.}\left(\bar{x}_{-}^{j}\right)\right) .
$$

Proof. For a given finite sequence $\left\{x^{i}\right\}_{i=0}^{m}$, choose the first pair of points of the subsequence to be $x^{0}$ and $x^{1}$.

We proceed to choose the next pair of points. Consider the closed ball $\bar{B}_{\alpha_{1} d\left(x^{0}, x^{1}\right) / 2}\left(\bar{x}_{+}^{0}\right)$, we choose $n_{2}>0$ to be the smallest integer such that $x^{n_{2}} \in$ $\bar{B}_{\alpha_{1} d\left(x^{0}, x^{1}\right) / 2}\left(\bar{x}_{+}^{0}\right)$ and we choose $n_{3}>0$ to be the largest integer such that $x^{n_{3}} \in$ $\bar{B}_{\alpha_{1} d\left(x^{0}, x^{1}\right) / 2}\left(\bar{x}_{+}^{0}\right) \cdot n_{2}$ and $n_{3}$ are the same if $\bar{B}_{\alpha_{1} d\left(x^{0}, x^{1}\right) / 2}\left(\bar{x}_{+}^{0}\right)$ contains only one point of the original sequence besides $x^{1}$. If this happens, we stop the selection process. If $\bar{B}_{\alpha_{1} d\left(x^{0}, x^{1}\right) / 2}\left(\bar{x}_{+}^{0}\right)$ contains no points other than $x^{1}$, we also end the selection process.

This finishes the first step in the selection process. At this point, we drop all the points in between $x^{n_{2}}$ and $x^{n_{3}}$ from the original sequence. The reason for doing this is that in the final perturbation we will push the point $x^{n_{3}}$ towards $x^{n_{2}}$, any points in between will not present any interference.

For the points $x^{n_{2}}$ and $x^{n_{3}}$ thus chosen, we can similarly define $\bar{x}^{1}=\left(x^{n_{2}}+\right.$ $\left.x^{n_{3}}\right) / 2$ and we can define $\bar{x}_{-}^{1}$ and $\bar{x}_{+}^{1}$ similar to $\bar{x}_{-}^{0}$ and $\bar{x}_{+}^{0}$ defined before. Our selection process bifurcates into two parts: one with $x^{n_{2}}$ and the one with $x^{n_{3}}$. We take $x^{n_{2}}$ first, $x^{n_{3}}$ will be dealt in a similar way.

Now we consider the closed ball $\bar{B}_{\alpha_{1} d\left(x^{n_{2}}, x^{n_{3}}\right) / 2}\left(\bar{x}_{-}^{1}\right)$. Let $i_{1}$ and $i_{2}$ be the smallest and respectively largest integer such that $x^{i_{1}}, x^{i_{2}} \in \bar{B}_{\alpha_{1}} d\left(x^{n_{2}}, x^{\left.n_{3}\right) / 2}\left(\bar{x}_{-}^{1}\right)\right.$. As $x^{n_{2}+1}, x^{n_{2}+2}, \ldots, x^{n_{3}-1}$ are dropped from consideration, we have three different situations: (1) $i_{1} \leqq i_{2}<n_{2}$; (2) $i_{1}<n_{2}<n_{3}<i_{2}$; and (3) $n_{3}<i_{1} \leqq i_{2}$. In all three cases, we choose $n_{4}=i_{1}$ and $n_{5}=i_{2}$ and drop all points in between $n_{4}$ and $n_{5}$ from future considerations. We point out that, for the second case, $n_{2}$ and $n_{3}$ are dropped from the previously chosen pairs.

We consider the second part of the selection process. If it is case (2) above, since $x^{n_{3}}$ has already been eliminated, we will not do anything further with respect to $x^{n_{3}}$. In all other cases, we consider the closed ball $\bar{B}_{\alpha_{1}} d\left(x^{n_{2}}, x^{n_{3}}\right) / 2\left(\bar{x}_{+}^{1}\right)$. Again, let $i_{1}$ and $i_{2}$ be the smallest and respectively largest integer such that $x^{i_{1}}, x^{i_{2}} \in \bar{B}_{\alpha_{1} d\left(x_{2}, x^{n_{3}}\right) / 2}\left(\bar{x}_{+}^{1}\right)$. This time, we have more cases for the position of $i_{1}$ and $i_{2}$. In general, if no previously chosen points fall in between $i_{1}$ and $i_{2}$, we let $n_{6}=i_{1}$ and $n_{7}=i_{2}$. 
However, if any previously chosen pairs fall in between $i_{1}$ and $i_{2}$, we still let $n_{6}=i_{1}$ and $n_{7}=i_{2}$. By eliminating all points in between $x^{n_{6}}$ and $x^{n_{7}}$, we have eliminated those previously chosen pairs.

To end the second step of the selection process, we rename these selected pairs so that they appear in ascending order.

We proceed by induction. In each of the following steps, we choose these pairs newly created in the previous step. Let $x^{n_{2 j}}$ and $x^{n_{2 j+1}}$ be one of such pairs. We define $\bar{x}^{j}, \bar{x}_{-}^{j}$ and $\bar{x}_{+}^{j}$. Then we first find a new pair in the closed ball $\bar{B}_{\alpha_{1} d\left(x^{n}{ }_{2 j}, x^{\left.n_{2 j+1}\right) / 2}\right.}\left(\bar{x}_{-}^{1}\right)$. We then eliminate all the points in the sequence between this new pair. If $x^{n_{2 j+1}}$ is not eliminated, we then go to choose a new pair in the closed

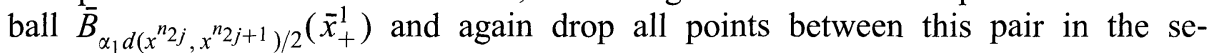
quence. Then we go on to the other pairs newly created in the previous step, if they are not eliminated yet. In the process, some of the closed balls may contain no or just one point of the sequence, we then end the selection process for these pairs. After each step, we rename all the remaining pairs in ascending order.

Since there are only a finite number of points in the sequence, the selection process ends in a finite number of steps.

This proves the lemma.

We have the following simple lemma.

Lemma 6. Let $\alpha_{1}, \sqrt{\frac{4 n-1}{4 n}}<\alpha_{1}<1$, be the number chosen previously. There exist positive numbers $0<\alpha_{2}<1$ and $\tau \geqq 1$ depending only on $\alpha_{1}$ and the dimension of $\mathbb{R}^{n}$ such that for any two distinct points $x^{0}, x^{1} \in \mathbb{R}^{n}$, there is a closed rectangle $D_{x^{*}}\left(\xi_{1}, \xi_{2}, \ldots, \xi_{n}\right)$, for some $x^{*} \in \mathbb{R}^{n}$ and $n$-tuple of positive real numbers $\left(\xi_{1}, \xi_{2}, \ldots, \xi_{n}\right)$ such that

1. $D_{x^{*}}\left(\xi_{1}, \xi_{2}, \ldots, \xi_{n}\right) \subset \bar{B}_{\alpha_{1} d\left(x^{0}, x^{1}\right) / 2}\left(x_{-}^{0}\right) \cup \bar{B}_{\alpha_{1} d\left(x^{0}, x^{1}\right) / 2}\left(x_{+}^{0}\right)$, where $x_{+}^{0}$ and $x_{-}^{0}$ are the points defined as before;

2. $x^{1}, x^{2} \in \alpha_{2} D_{x^{*}}\left(\xi_{1}, \xi_{2}, \ldots, \xi_{n}\right)$;

3. $\xi_{i} / \xi_{j} \leqq \tau$, for all $i, j=1,2, \ldots, n$.

Proof. The proof is easy. First observe that the choice of the rectangles and $\alpha_{2}$ is independent of $d\left(x^{0}, x^{2}\right)$, hence we may assume that $d\left(x^{0}, x^{1}\right)=1$. For any two points $x^{0}, x^{1} \in \mathbb{R}^{n}$, let $D_{1}$ be the smallest rectangle (with sides parallel to coordinate planes) that covers both $x^{0}$ and $x^{1}$. By the definition of $\bar{x}_{-}^{0}, \bar{x}_{+}^{0}$ and $\alpha_{1}$, the union of the balls

$$
B_{\alpha_{1} d\left(x^{0}, x^{1}\right) / 2}\left(\bar{x}_{-}^{0}\right) \cup B_{\alpha_{1} d\left(x^{0}, x^{1}\right) / 2}\left(\bar{x}_{+}^{0}\right)
$$

contains $D_{1}$ in its interior. The rectangle $D_{1}$ may be degenerate with the lengths of one or more sides equal to zero, if some of the components of $x^{0}-x^{1}$ are zero. If this happens, we enlarge the rectangle $D_{1}$ a little bit to make it non-degenerate and still contained in the interior of the union of the two balls. Finally, we choose $D_{x^{*}}\left(\xi_{1}, \xi_{2}, \ldots, \xi_{n}\right)$ to be the largest rectangle contained in the union of the two closed balls that has $D_{1}$ in its interior. This way, for each pair $x^{0}, x^{1}$, we find $\alpha_{2}, \tau$ and a rectangle $D_{x^{*}}\left(\xi_{1}, \xi_{2}, \ldots, \xi_{n}\right)$ that satisfy the lemma. To get a uniform $\alpha_{2}$ and $\tau$, we just take the suprema of all the $\tau$ and $\alpha_{2}$. By the compactness of the choices of $x^{0}, x^{1}$ and local continuity of $\tau$ and $\alpha_{2}$, such suprema exist and $\tau<\infty, \alpha_{2}<1$.

This proves the lemma. 
Lemma 7. There exist real numbers $0<\alpha_{3}<1, \tau \geqq 1$ and a positive integer $N^{*}$ such that for any $n$-tuple $\left(a_{1}, a_{2}, \ldots, a_{n}\right)$ of positive numbers, any sequence $\left\{x^{i}\right\}_{i=0}^{m}$ in $\mathbb{R}^{n}$ with $d\left(x^{i}, x^{0}\right) \geqq d\left(x^{1}, x^{0}\right)$ for all $i>1$, there exists a finite subsequence $\left\{x^{n_{i}}\right\}_{i=0}^{2 j^{*}+1}, n_{2 i} \leqq n_{2 i+1}$ for $i=0,1, \ldots, j^{*}$ and $n_{i}<n_{j}$ for $j>i+1$, with $x^{0}$ and $x^{1}$ being the first two points, such that there exist $j^{*}$ number of rectangles $D_{1}, D_{2}, \ldots, D_{j^{*}}$ satisfying the following properties, where for simplicity, we denote $D_{i}=D_{\bar{x}^{i}}\left(\xi_{1}^{i}, \xi_{2}^{i}, \ldots, \xi_{n}^{i}\right)$ for some $\bar{x}^{i} \in \mathbb{R}^{n}$ and some $n$-tuple of positive numbers $\left(\xi_{1}^{i}, \xi_{2}^{i}, \ldots, \xi_{n}^{i}\right)$ :

1. $\left(\xi_{j}^{i} / a_{j}\right) /\left(\xi_{l}^{i} / a_{l}\right) \leqq \tau$, for all $i=1,2, \ldots, j^{*}$ and $j, l=1,2, \ldots, n$.

2. $x^{n_{2 i}}, x^{n_{2 i+1}} \in \alpha_{3} D_{i}$, for $i=0,1,2, \ldots, j^{*}$.

3. If $n_{2 j+1}<i<n_{2 j+2}$ for some $j=0,1, \ldots, j^{*}$, then $x^{i} \notin \bigcup_{j=0}^{j^{*}} D_{j}$.

4. Let $\mathscr{S}$ be a subset of $\left\{0,1, \ldots, j^{*}\right\}$ with size $N^{*}$. Then for any such $\mathscr{S}$,

$$
\bigcap_{i \in \mathscr{S}} D_{i}=\emptyset \text {. }
$$

Or roughly, any point in $\mathbb{R}^{n}$ is at most covered by $N^{*}$ number of $D_{i}$ 's.

Proof. We first prove the lemma for the case where the $n$-tuple of positive real numbers $\left(a_{1}, a_{2}, \ldots, a_{n}\right)$ are all equal to one. Let $\left\{x^{i}\right\}_{i=0}^{m}$ in $\mathbb{R}^{n}$ be a sequence of points with $d\left(x^{i}, x^{0}\right) \geqq d\left(x^{1}, x^{0}\right)$ for all $i>1$. By Lemma 5 , there exists a subsequence $\left\{x^{n_{i}}\right\}_{i=0}^{2 j^{*}+1}$ satisfying the list of properties in Lemma 5 .

We apply Lemma 6 to each pair in the selected subsequence. Let $x^{n_{2 i}}, x^{n_{2 i+1}}$ for $i=0,1, \ldots, j^{*}$ be a pair of points in the subsequence. By Lemma 6 , there exist a positive integer $0<\alpha_{2}<1$ independent of these points, and a closed rectangle $D_{i}=D_{\bar{x}^{i}}\left(\xi_{1}^{i}, \xi_{2}^{i}, \ldots, \xi_{n}^{i}\right)$ such that

$$
D_{i} \subset B_{\alpha_{1} d\left(x^{n} 2 i, x^{n} n_{2 i+1}\right) / 2}\left(\bar{x}_{+}^{i}\right) \cup B_{\alpha_{1} d\left(x^{n} 2 i, x^{n} 2 i+1\right) / 2}\left(\bar{x}_{-}^{i}\right)
$$

and $x^{n_{2 i}}, x^{n_{2 i+1}} \in \alpha_{2} D_{i}$, where $\xi_{j}^{i} / \xi_{l}^{i} \leqq \tau$, for all $i=0,1,2, \ldots, j^{*}$ and $j, l=1,2, \ldots, n$. $\tau$ is given in Lemma 6 .

Let $D_{0}, D_{1}, \ldots, D_{j^{*}}$ be the closed rectangles thus chosen. We point out that some of these rectangles may be degenerate and contain just one point (for some pairs with just one distinct point). For these single point rectangles, we may choose any value we like for the ratio $\frac{\xi_{i}}{\xi_{j}}$. In this way we have a sequence of rectangles $D_{1}, D_{2}, \ldots, D_{j^{*}}$ satisfying the first three properties in the conclusion of the lemma, with $\alpha_{3}=\alpha_{1}$ and $\tau$ given in Lemma 6 .

To get property 4 of Lemma 7 , we first observe that in each step of our construction, the radii of all the closed balls are shrunk by at least a factor of $\alpha_{1}<1$. This implies that in the following steps, the radii of the closed balls decrease faster than a geometric series. We consider a rectangle $D_{i}$. If we shrink $D_{i}$ 's by a small factor of $\left(1+\alpha_{1}\right) / 2$ to obtain $D_{i}^{\prime}=\left(1+\alpha_{1}\right) / 2 D_{i}$, then we have $x^{n_{2 i}}, x^{n_{2 i+1}} \in \alpha^{\prime} D_{i}^{\prime}$, with $\alpha^{\prime}=2 \alpha_{1} /\left(1+\alpha_{1}\right)<1$. Moreover, there exists a positive constant $c_{1}>0$, independent of $D_{i}$, such that if $\xi_{j}^{1} \leqq c_{1} \xi_{i}^{1}$, then $D_{j} \cap D_{i}^{\prime}=\emptyset$. This implies that $D_{i}^{\prime}$ can only have intersections with $D_{j}=D_{\bar{x}^{j}}\left(\xi_{1}^{j}, \xi_{2}^{j}, \ldots, \xi_{n}^{j}\right)$ with $\xi_{1}^{j}>c_{1} \xi_{1}^{i}$. However, with the condition of $\xi_{l}^{j} / \xi_{k}^{j} \leqq \tau$ for all $j=0,1, \ldots, j^{*}$ and $l, k=1,2, \ldots, n$ and the way all boxes are defined, we have that the number of points in each box is uniformly bounded, therefore there exists a positive integer $N^{*}, N^{*}$ depending only on $\alpha_{1}, \tau$ and $n$, such that $D_{i}^{\prime}$ has intersections with at most $N^{*}$ number of other boxes. 
Let $\alpha_{3}=\alpha^{\prime}=\left(2 \alpha_{1}\right) /\left(1+\alpha_{1}\right)$ and replace $D_{i}$ with $D_{i}^{\prime}$; we obtain property 4 in the lemma.

This proves Lemma 7 for the case where $a_{i}=1$ for all $i=1, \ldots, n$. The general case reduces to the above case with a rescaling of each coordinate axis by $a_{1}, a_{2}, \ldots, a_{n}$ respectively.

This completes the proof of Lemma 7.

\section{Proof of Theorem 1}

In this section, we prove Theorem 1.

Let $M^{n}$ be a compact $n$-dimensional manifold with a symplectic or volume structure $\omega$. Let $\phi$ be a diffeomorphism that preserves $\omega$. Let $p$ be a hyperbolic periodic point of period $k$ for $\phi$ and let $q$ be a point in the unstable manifold of $p$. By a small perturbation, we may assume that all eigenvalues of

$$
\left.d\left(\phi^{k}\right)\right|_{T_{p} M^{n}}: T_{p} M^{n} \rightarrow T_{p} M^{n}
$$

are simple and away from unit circle.

First, we prove Theorem 1 for the case where $p$ is a fixed point of $\phi$.

By Lemma 2, we may assume that $q$ is recurrent. Therefore there exists a point $x^{0} \in W_{\phi}^{s}(p)$ such that $x^{0}$ is in the $\omega$-limit set of $q$, i.e., there exists a sequence of positive integer $\left\{n_{i}\right\}_{i=1}^{\infty}$ such that $\phi^{n_{i}}(q) \rightarrow x^{0}$. Fix a small neighborhood of $x^{0}, W$. For any $\eta>0$ small, there exists a $j^{\prime}$ such that $d\left(x^{0}, \phi^{j^{\prime}}(q)\right)<\eta$. We may assume that for $0 \leqq i<j^{\prime}, d\left(x^{0}, \phi^{i}(q)\right)>d\left(x^{0}, \phi^{j^{\prime}}(q)\right)$. Let $\left\{\phi^{j^{\prime}-i}(q)\right\}_{i=0}^{j^{\prime}}$ be a segment of the orbit of $q$ in reverse order. For the fixed neighborhood $W$ of $q$, starting with $\phi^{j^{\prime}}(q)$, we obtain a subsequence of intersections of the sequence $\left\{\phi^{j^{\prime}-i}(q)\right\}_{i=0}^{j^{\prime}}$ with $W$. We rename the subsequence $\left\{x^{i}\right\}_{i=1}^{m}$ for some integer $m$. Note that $x^{1}=\phi^{j^{\prime}}(q)$.

In what follows, we are only concerned with a small neighborhood of $p \in M^{n}$. We use local coordinates. We choose a coordinate chart $\psi: \mathbb{R}^{n} \rightarrow B_{r}(p) \subset M^{n}$ for some small $r$ such that $\psi(O)=p$ and $f=\psi^{-1} \phi \psi$ takes the following form: $f(\mathbf{x})=\psi^{-1} \phi \psi(\mathbf{x})=A \mathbf{x}+o(\|\mathbf{x}\|)$, where $A$ is in Jordan canonical form and has only simple eigenvalues.

Fix a given $\varepsilon>0$ small. Our goal is to do a $C^{1} \varepsilon$ small perturbation with support in $D_{0}, D_{1}, \ldots, D_{j^{*}}$ and their images so that $f^{N}\left(x^{n_{2 j+1}}\right)$ is pushed to $f^{N}\left(x^{n_{2 j}}\right)$ for some $N$ and for all $j=0,1, \ldots, j^{*}$. Since $D_{0}, D_{1}, \ldots, D_{j^{*}}$ contains no points $x^{i}$ with $n_{2 j}<i<n_{2 j+1}$, this perturbation process eliminates all the intermediate intersections between $x^{n_{2 j}}$ and $x^{n_{2 j+1}}$, and connects the orbit of $q$ with the orbit of $x^{0}$, creating homoclinic points. Since we may still have interventions between the selected pairs, the perturbation has to be done in a careful way. In particular, we have to shrink $D_{i}$ 's further to leave room for interventions.

Let $0<\alpha_{3}<1$ and $N^{*}$ be given by Lemma 7. Let $c$ be the positive number given by the perturbation lemma. Let $\alpha=\left(\alpha_{3}+\left(1-\alpha_{3}\right) c \varepsilon^{N^{*}}\right) / \alpha_{3}$. By Lemmas 3 and 4 , there exist an integer $N$ and an $n$-tuple $\left(a_{1}, a_{2}, \ldots, a_{n}\right)$ of positive numbers and $\delta>0$, depending only on eigenvalues of $A, \varepsilon$ and $\alpha$ ( $\delta$ also depends on $f$ ), such that the conclusions of Lemmas 3 and 4 hold in the domain $B_{\delta}(O)$.

By replacing $x^{0}$ above with one of its iterations, we may assume that $x^{0} \in B_{\delta}(O)$. (Here for simplicity of notation, we identify points on $M^{n}$ with points on $\mathbb{R}^{n}$ ). For an $\eta>0$, let $\left\{x^{i}\right\}_{i=0}^{m} \subset \mathbb{R}^{n}$ be the sequence selected above. For the $n$-tuple 
$\left(a_{1}, a_{2}, \ldots, a_{n}\right)$ chosen above by Lemmas 3 and 4 , by Lemma 7 , we find a subsequence $\left\{x^{n_{i}}\right\}_{i=0}^{j^{*}}$ together with a sequence of boxes $D_{1}, D_{2}, \ldots, D_{j^{*}}$ satisfying the properties listed in Lemma 7.

By making $\eta$ small, we may assume that each box $D_{i}$ has size $\left(\xi_{1}^{i}, \xi_{2}^{i}, \ldots, \xi_{n}^{i}\right)$, and the sequence of rectangular boxes and their iterates under $f$ up to $N^{\text {th }}$ are all in $B_{\delta}(O)$ and the origin $O \notin D_{i}$.

First assume that $p$ is a fixed point, periodic points with period larger than one will be dealt with later. We are now ready to do a sequence of perturbations to prove Theorem 1 for this case. We consider all the boxes $\left(\alpha_{3} / \alpha\right) D_{0},\left(\alpha_{3} / \alpha\right) D_{1}, \ldots,\left(\alpha_{3} / \alpha\right) D_{j^{*}}$ and we do the perturbation simultaneously in each box. In each step, we try to push $x^{n_{2 j+1}}$ towards $x^{n_{2 j}}$.

As in the proof of Lemma 3, in the first $N_{1}$ iterates of $f$, we do the perturbations in the first coordinate direction. In each iterate, we push the amount $(1-\alpha) c \varepsilon \xi_{1}^{i} / \alpha$ and the support needed for each push is $(1-\alpha) \xi_{1}^{i} / \alpha$. We remark that, by our choice of $\alpha,(1-\alpha)$ is an extremely small number $\left(\sim \varepsilon^{N^{*}}\right)$. The support required in each push is very small and is far away from the boundary of $D_{i}$. In this way, if interventions between different pairs occur (i.e., when their supports intersect), we have enough room left for larger perturbation. If there are no interventions between pairs, we go onto the next coordinate and continue the perturbation.

The problem arises when interventions between pairs arise. However, by Lemma 7, on a given $D_{i}$, at most $N^{*}$ number of other boxes may present interventions at any given iterate.

Let's suppose at some step the perturbation in $D_{i}$ interferes with the perturbation in $D_{j}, i<j$. This implies that the supports of two different pushes have common points. In this case, we will push $x^{n_{2 j+1}}$ towards $x^{n_{2 i}}$. This requires a larger support and this support is provided in either $D_{i}$ or $D_{j}$, whichever is larger, because of our choice of $\alpha$. After this step, we drop all the boxes $D_{i+1}, D_{i+2}, \ldots, D_{j}$ and continue the perturbation process as before. In the end, we have eliminated all the intermediate intersections between $x^{n_{2 i}}$ and $x^{n_{2 j+1}}$.

There are other forms of intervention between different pairs. One is simultaneous interventions between many different boxes. In this case, we push the point with largest index towards the point with smallest index and eliminate all the intermediate points. In this case, the support is in the largest box. Another form of intervention is when we have interventions between two boxes, $D_{i}$ and $D_{j}$. However, if we try to push $x^{n_{2 j+1}}$ towards $x^{n_{2 i}}$, intervention with $D_{k}$ occurs because a larger support is needed. This and similar cases can be all dealt in the same way: find all possible interventions (at most $N^{*}$ ) and do the perturbation to push the point with largest index towards the point with smallest index. This is possible because we have chosen the support of each push extremely small. The number of iterates needed for the perturbation is very large.

This finishes the proof of Theorem 1 in the case where $p$ is a fixed point of $\phi$. The case where $p$ is a hyperbolic periodic point of period $k, k>1$, is very much similar. Instead of considering $\phi$, we consider $\phi^{k}$ and apply the series of perturbations to $\phi^{k}$. We obtain a diffeomorphism $G \in \operatorname{diff}_{\omega}^{1}\left(M^{n}\right)$, with $\left\|G-\phi^{k}\right\|_{C^{1}}<N^{*} \varepsilon$ and $\operatorname{Support}\left(G-\phi^{k}\right) \subset B_{\delta}(p)$, such that $q$ is a homoclinic point for $G$. We may assume that $\phi^{i}\left(B_{\delta}(p)\right) \cap B_{\delta}(p)=\emptyset$ for $i=1,2, \ldots, k-1$. Let $g=G \phi^{-k} \phi$, then $\|g-\phi\|_{C^{1}}<N^{*} \varepsilon$ and $g^{k}=G$. We conclude that $g$ is the required perturbation of $\phi$.

This proves Theorem 1. 


\section{Proof of Theorem 2 and Theorem 3}

In this section, we first give a proof of Theorem 2 .

Let $k \geqq 1$ be some positive integer. From Robinson's theorems [9], there is an open and dense set $\mathscr{B}_{k} \subset \operatorname{diff}_{\omega}^{1}\left(M^{n}\right)$ such that for each $\phi \in \mathscr{B}_{k}$, all the periodic points of $\phi$ with period less than or equal to $k$ are nondegenerate and thus there are only finitely many of these periodic points. Let $p_{i}^{k}, i=1,2, \ldots, m_{k}$ be the hyperbolic periodic points with respect to $\phi$. In a connected component of $\mathscr{B}_{k}$ containing $\phi, p_{i}^{k}$ varies continuously with respect to $\phi \in \mathscr{B}_{k}$. For each $p_{i}^{k}$, let $W_{\phi, ~ l o c}^{s}\left(p_{i}^{k}\right)$ and $W_{\phi, l o c}^{u}\left(p_{i}^{k}\right)$ be a local stable manifold and a local unstable manifold of $p_{i}^{k}$ with respect to $\phi$. Let

$$
E_{\phi}^{s}\left(p_{i}^{k}\right)=\operatorname{Closure}\left(\phi^{-1}\left(W_{\phi, \mathrm{loc}}^{s}\left(p_{i}^{k}\right)\right) \backslash W_{\phi, \mathrm{loc}}^{s}\left(p_{i}^{k}\right)\right)
$$

and

$$
E_{\phi}^{u}\left(p_{i}^{k}\right)=\operatorname{Closure}\left(\phi\left(W_{\phi, \mathrm{loc}}^{u}\left(p_{i}^{k}\right)\right) \backslash W_{\phi, \mathrm{loc}}^{u}\left(p_{i}^{k}\right)\right) .
$$

$E_{\phi}^{s}\left(p_{i}^{k}\right)$ and $E_{\phi}^{s}\left(p_{i}^{k}\right)$ are called the fundamental domains of the stable manifold and unstable manifold of $p_{i}^{k}$.

For any positive integer $j>0$, let $\left\{U_{i}\right\}$ be a finite open cover of $M^{n}$ with diameter less than $1 / j$. Let $\mathscr{B}_{k, 1 / j} \subset \mathscr{B}_{k} \subset \operatorname{diff}_{\omega}^{1}\left(M^{n}\right)$ be a subset with the following property: for each map $\phi \in \mathscr{B}_{k, 1 / j}$ and each hyperbolic periodic point $p_{i}^{k}$ with period less than or equal to $k$, with respect to $\phi$, if

$$
U_{i} \cap\left(E_{\phi}^{s}\left(p_{i}^{k}\right) \cup E_{\phi}^{u}\left(p_{i}^{k}\right)\right) \neq \emptyset
$$

for any $i$, then there exists a transversal homoclinic point in $U_{i} \cap\left(E_{\phi}^{s}\left(p_{i}^{k}\right) \cup\right.$ $\left.E_{\phi}^{u}\left(p_{i}^{k}\right)\right)$, i.e., the transversal homoclinic points in fundamental domains of the stable and unstable manifolds are at most separated by a distance of $1 / j$ for maps in $\mathscr{B}_{k, 1 / j}$. It is obvious that the set $\mathscr{B}_{k, 1 / j}$ is open. Theorem 1 shows that $\mathscr{B}_{k, 1 / j}$ is also dense. Thus $\mathscr{B}_{k, 1 / j}$ is an open and dense set in $\operatorname{diff}_{\omega}^{1}\left(M^{n}\right)$. Take

$$
\mathscr{B}=\bigcap_{k=1}^{\infty} \bigcap_{j=1}^{\infty} \mathscr{B}_{k, 1 / j} \subset \operatorname{diff}_{\omega}^{1}\left(M^{n}\right) \text {. }
$$

Then for any $\phi \in \mathscr{B}$, all periodic points of $\phi$ are nondegenerate and for each hyperbolic periodic point of $\phi$, the transversal homoclinic points are dense in some fundamental domains of its stable and unstable manifolds. Since $\mathscr{B}$ is a countable intersection of open and dense sets, it is a residual set.

This proves Theorem 2 .

Since all lemmas except Lemma 2 work for general diffeomorphisms, the proof of Theorem 3 follows from the proof of Theorem 1 with some small modifications. Let

$$
q \in \operatorname{closure}\left(W_{\phi}^{s}(p)\right) \cap W_{\phi}^{u}(p) \backslash\{p\} .
$$

There exists a point $q^{\prime} \in W_{\phi}^{s}(p)$ such that for any $\delta>0$, there is a point $x$ and integer $m>0$ such that $d(x, q)<\delta$ and $d\left(f^{m}(x), q^{\prime}\right)<\delta$. Now the theorem follows with applying the connecting procedure at both $q$ and $q^{\prime}$.

We end this paper by remarking that if the condition in Theorem 3 is replaced by

$$
q \in \operatorname{closure}\left(W_{\phi}^{s}(p)\right) \cap \operatorname{closure}\left(W_{\phi}^{u}(p)\right) \backslash\{p\},
$$


and assume that $q$ is not a periodic point, then the theorem is still true. However, we have to rely on these properties on arbitrary sequences of linear isomorphisms obtained by Pugh in his closing lemma.

\section{References}

1. Hayashi, S.: A connecting lemma. In Preparation

2. Newhouse, S.: Quasi-elliptic periodic points in conservative dynamical systems. Am. J. Math. 99, 1061-87 (1977)

3. Oliveira, F.: On the generic existence of homoclinic points. Ergod. Th. \& Dynam. Sys. 7, 567-595 (1987)

4. Pixton, D.: Planar homoclinic points. J. Differ. Eqs. 44, 365-382 (1982)

5. Poincare, H.: Les Methodes Nouvelles de la Mécanique Celeste. Tome II, 1899

6. Pugh, C.: The closing lemma. Am. J. Math. 89, 956-1021 (1967)

7. Pugh, C.: The $C^{1}$ connecting lemma. J. Dynam. \& Diff. Eqs. 4, No. 4, 545-553 (1992)

8. Pugh, C., Robinson, C.: The $C^{1}$ closing lemma, including Hamiltonians. Ergod. Th. \& Dynam. Sys. 3, 261-313 (1983)

9. Robinson, C.: Generic Properties of Conservative Systems, I, II. Am. J. of Math. 92, 562603, 897-906 (1970)

10. Robinson, C.: Closing stable and unstable manifolds on the two-sphere. Proc. Am. Math. Soc. 41, 299-303 (1973)

11. Takens, F.: Homoclinic points in conservative systems. Invent. Math. 18, 267-292 (1972)

Communicated by S.-T. Yau 
